Pensamiento Crítico Vol.17. N¹, pp. 31-45

\title{
Una apreciación a la comercialización de la producción minero metalúrgica en el Perú, periodo 1970-2010.
}

\author{
Juan José Ospino Edery
}

\section{RESUMEN}

El presente estudio desarrolla un particular análisis que abarca cuatro periodos sobre la evolución e implicancias de la actividad minero metalúrgica y su comercialización.

El primer periodo normado y regulado por el código de minería de 1950, el cual orientó e impulsó de manera liberal la inversión en minería de capitales principalmente norteamericanos, un segundo periodo considerado entre los años (1968-1980), etapa durante la cual el estado asumió el rol de promotor, ejecutor y líder de la actividad minero metalúrgica y su comercialización de forma monopólica en cumplimiento de la Ley general de minería 18880.

Un tercer periodo desde 1980-1990, el cual involucra tanto al segundo gobierno del presidente Femando Belaunde como al primer gobierno de Alan García, etapa durante la cual se mantuvo el papel del estado en lo referente a su accionar como promotor y líder de la actividad minero metalúrgica, variando únicamente su rol monopólico en la comercialización de la producción minera.

Un cuarto y último periodo que se encuentra vigente, caracterizado por la privatización de las empresas mineras que eran de propiedad del estado con 


\section{Pensamiento Crítico Vol.I7. No I}

lo cual se extingue su accionar en la compraventa de minerales, concentrados, fundidos y refinados, regresando en la práctica a esquemas de explotación minera y comercialización similares a las fomentadas por el código de minería de los años cincuenta.

Con la aplicación en extremo de políticas económicas de promoción a la inversión en minería de carácter liberal, no se tiene un control/fiscalización de la real valorización de la producción minero metalúrgica y su consecuente comercialización, lo cual estaría permitiendo prácticas de subvaluación y sobrevalorización (transferencia de precios), así mismo se limita la generación de ahorro interno dirigido a inversiones orientadas a la búsqueda de un mayor valor agregado del producto minero, dado que la mayor parte de la inversión minera obedece y depende de capitales foráneos.

Palabras claves: Comercialización, producción de concentrados mineros, exportación de concentrados sobrevalorización, subvaluación, refinados mineros, maquila, precio de metal.

\section{ABSTRACT}

This study develops a particular diagnosis that covers four periods of the evolution and implications of mining and metallurgical activity and marketing.

The first period regulated by de mining code of 1950, which guided and impetus so liberal mining investment capital mainly American, a second period by the years 1968-1980, period during which the state assumed the role of promoter, performer and leader of the mining and metallurgical activity and marketing of monopoly in compliance with the General Mining Law 18880.

A third period from 1980-1990, involving both the second administration of President Fernando Belaunde as first government of Alan Garcia, a stage during which remained the state's role in relation to activity as a promoter and leader of mining activity, metal, varying only their role in the marketing monopoly of mining production.

A fourth and final period is in effect, characterized by privatization of mining companies that were owned by the state thus extinguished their actions in the sale of ores, concentrates, melted and refined in practice back schemes mining and marketing similar to those promoted by the mining code of the fifties.

Keywords: marketing, production of mineral concentrates, export of concentrates overvaluation, undervaluation, refined mining, manufacturing, metal price. 


\section{Juan José Ospino Edery}

\section{Introducción}

Trabajos previos o contemporáneos sobre la problemática social económica de la comercialización de la producción minero metalúrgica son limitados al interior de la UNMSM, con la excepción de trabajos propios que abarcan temas relacionados.

Podemos aseverar que una situación similar se observa en lo que respecta a la bibliografía publicada en el ámbito nacional, a pesar y dada la relevancia que tiene la actividad minera, donde el quehacer de la comercialización de la producción minero Metalúrgica se constituye en la razón de la Inversión Minera y la llave para propiciar y sustentar un genuino desarrollo económico.

\section{Marco referencial}

Cuando nos referimos a la Comercialización de la producción minero metalúrgica, la razón del negocio de la inversión minera, nos estamos refiriendo a las implicancias que tiene la misma para generar mayores ingresos a sus actores, superiores ingresos tributarios y la constitución de un mayor ahorro interno.

Mayores ingresos tributarios, al hacer transparente el verdadero valor de la producción minero metalúrgica exportable evitando la sobrevalorización o subvaluación dada la manipulación de las leyes para un mayor o menor pago de sus valores reales de mercado.

Poner en evidencia, el conocimiento de una actividad que pertenece al sector terciario el cual tiene importancia y desarrollo creciente en países de economías más avanzadas, Know how que como país dejamos de conocer y desarrollar al darse el proceso de privatización de la actividad comercializadora en los primeros años de gobierno del presidente Fujimori, luego de estar a la vanguardia.

La comercialización de la producción minero metalúrgica, implica de manera adicional a la compraventa interna-externa de minerales, concentrados, fundidos y refinados, el estudio del comportamiento de los principales mercado y su proyección mediante el uso de modelos econométricos, los mecanismos de determinación de los precios en las bolsas así como la generación de operaciones en las mismas (coberturas, opciones, arbitrajes etc.), el manejo de la negociación del flete marítimo y otros. 


\section{Pensamiento Crítico Vol.I7. No I}

Se considera a la Comercialización de la Producción Minero Metalúrgica, como capítulo integrante de lo que se ha denominado economía minera, materia que es estudiada como curso de postgrado o que la misma se constituye en una especialidad para una Maestría o un Doctorado en Universidades de países vecinos, aspecto que se encuentra descuidado y no reconocido por nuestras universidades, dada la implicancia económica de esta actividad

\section{Factores que intervienen en la determinación de los precios de los metales}

Frecuentemente vemos o escuchamos que los precios de los metales subieron o bajaron por diferentes factores, los cuales no dependen del comportamiento económico de nuestro país sino que los mismos están sujetos a determinadas causas que son importantes apreciar su evolución, dado que en las diferentes operaciones de compraventa interna y externa que realiza la pequeña, mediana o gran minería, ya sea de minerales, concentrados o refinados se utilizan fórmulas para su valoración (ver entrecomillado), las que tienen como referencia los precios que se determinan en las bolsas $\mathrm{u}$ otras organizaciones que cumplen una función similar ${ }^{1}$.

"Los concentrados de los metales principales se valorizan a los precios de los metales menos descuentos/deducciones por costos de tratamiento, pérdidas y otros factores. Las siguientes fórmulas permiten determinar el precio que se paga por los concentrados", ver tabla siguiente $\mathrm{N} .^{\circ} 1$.

Tabla No 1

Formulas y Variables para Valorizar Concentrados Minerales

Fórmula A: VC $=[M-D]\left[P . F-\left(R-+E^{\prime \prime}\right)\right]-(T-+E 2)-X+Y$

Fórmula $B: V C=[M-D][P$. F $]-[(T-+E)+T(P-P B)]-X+Y$

Donde :

VC: Valor del concentrado

M: Contenido de Metal

D : Deducciones por perdida metalúrgica

P : Precio del metal (cotización referencial utilizada)

1 Entre las Bolsas de Metales son las más reconocidas la Bolsa de Metales de Londres (LME) y la de Nueva York (COMEX/NYMEX). Y las Publicaciones American Metal Market. Metal Bulletin, Metals Week. 


\section{Juan José Ospino Edery}

F : Factor del precio

$\mathrm{R}$ : Maquila de refinación (Refining Charge)

E1 : Escalador de maquila de refinación

$\mathrm{T}$ : Maquila de tratamiento

E2 : Escalador de maquila de tratamiento

PB : Precio base referente al costo de tratamiento(fundición y/o refinación)

$\mathrm{T}$ : Factor de ajuste del costo de tratamiento

$\mathrm{X}$ : Deducciones por impurezas

Y : Créditos por subproductos

Fuente: Economía Minera - Alfredo Dammert Lira/Minpeco 1980/1990

Los precios de los minerales, concentrados y refinados más representativos para nuestra economía (cobre, plomo, plata, zinc, oro, llámense metales) son comúnmente el reflejo de las condiciones que imperan en cada momento en el mercado mundial. En el cual participan compradores y vendedores de toda magnitud, influenciando de manera decisiva en las fluctuaciones y la estabilidad de los precios.

Es así como los grandes compradores de los países industrializados (Estados Unidos, Japón, Alemania, Francia, Italia, España y otros de la Comunidad Económica Europea, y los llamados emergentes como China, India, Brasil etc) juegan un papel decisivo en el funcionamiento del mercado mundial donde los efectos de las situaciones políticas, económicas, financieras que atraviesan estos países, repercuten en los precios de los metales y en las expectativas futuras en cuanto a las cotizaciones de los mismos.

El mercado de los metales (refinados) tiene un funcionamiento complejo, tal es así que en la fijación de su precio es indudable que se tenga en cuenta tanto el volumen ofertado por parte de los productores, y la demanda por parte de los consumidores (industriales), que encuentran en el mercado un precio que les permite perfeccionar un negocio de compra y venta; pero no siempre los precios que rigen el mercado reflejan la situación tanto de la oferta y demanda.

Cabe resaltar que el mercado de concentrados para nuestro país resulta el de mayor envergadura dado que bajo esta calidad son los mayores volúmenes que exportamos a fundiciones y refinerías que son en número mucho menor que los compradores de refinados (industriales). 


\section{Pensamiento Crítico Vol.17. No I}

Es notorio que los factores que afectan la fijación de los precios de los metales puedan darse por un exceso de producción del metal, el cual se hace sentir rápidamente en el mercado, igualmente un aumento violento en la demanda influye de inmediato en los precios que se registran. A los factores mencionados se suman otros que en gran parte influencian directamente en la generación de los precios al contado y a futuro, como por ejemplo: la existencia de metales en stock, en poder de los productores, comerciantes, consumidores y las bolsas de metales.

De manera adicional la calidad del comprador o vendedor, concediendo descuentos, premios de diversa índole, facilidades de pago, etc.

\section{Estructura de la Comercialización de la Producción Minero Metalúrgica, periodo 1950-1970.}

Flujograma de la Comercialización de la Producción Minero Metalúrgica

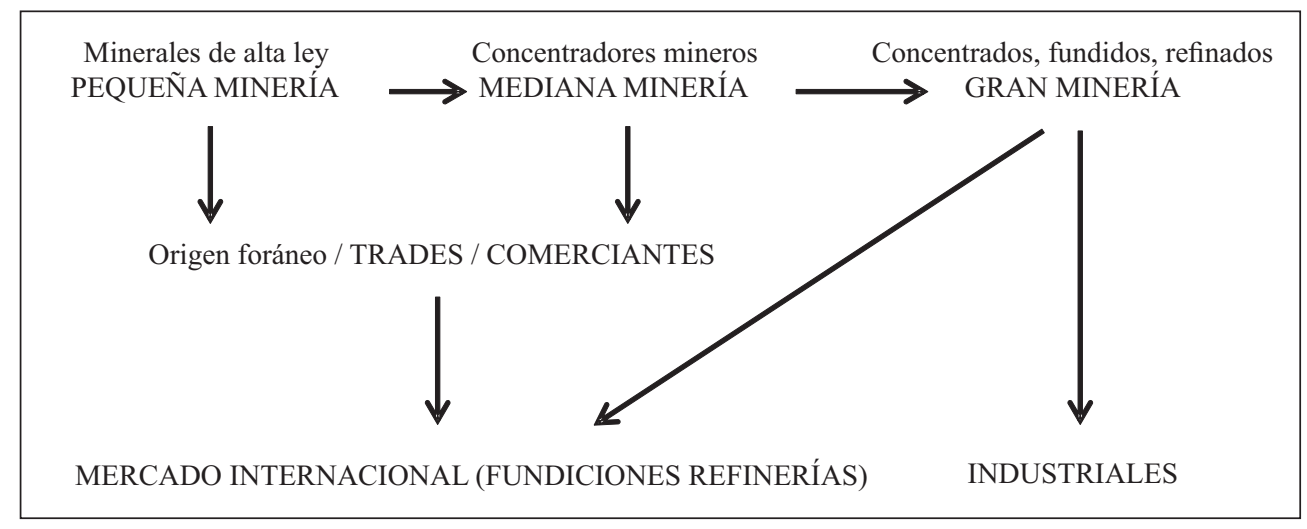

En este periodo la actividad minera estuvo regulada por el Código de Minería de 1950, el cual determinó una serie de dispositivos que tenían por finalidad crear las condiciones favorables para la realización de inversiones de origen foráneo, siendo las inversiones de los EE.UU. las que lideraron.

Dispositivos que se referían entre otros a: descuentos por el establecimiento de una reserva por agotamiento, liberación de todo derecho de importación, garantías tributarias, garantías para reexportar capitales y utilidades a la casa matriz, etc. 


\section{Juan José Ospino Edery}

En este contexto la explotación minera se incrementó en nuestro país, destacándose la zona de la sierra central, tanto por el accionar de empresas medianas como las pequeñas. Por otro lado, el desarrollo de la tecnología mediante una alta mecanización facilitó la explotación de reservas de cobre de baja ley mediante el método de tajo abierto, en los yacimientos mineros de Toquepala y Cuajone, y el inicio de operaciones para la explotación de hierro por parte de la Marcona Mining Company.

La comercialización de la producción minero metalúrgica era realizada por las mismas compañías, y a la vez existían empresas especializadas en dicha actividad, la mayoría de origen extranjero, destacando las empresas Minerco, Hoschild, Cerro Sales, Nachman, etc.

Se aprecia del flujograma anterior, que el estado no tenía mayor control ni fiscalización sobre la comercialización de la producción minero metalúrgica, situación que habría permitido actividades de subvaluación y sobrevalorización, que llevaba a sus actores a formas de evasión tributaria, así como una explotación no sostenible y de tremendo impacto ambiental de carácter irreversible para las poblaciones vecinas a los yacimientos mineros.

\section{Estructura de la Comercialización de la Producción Minero Metalúrgica, Periodo 1970-1980}

Flujograma de la Comercialización de la Producción Minero Metalúrgica

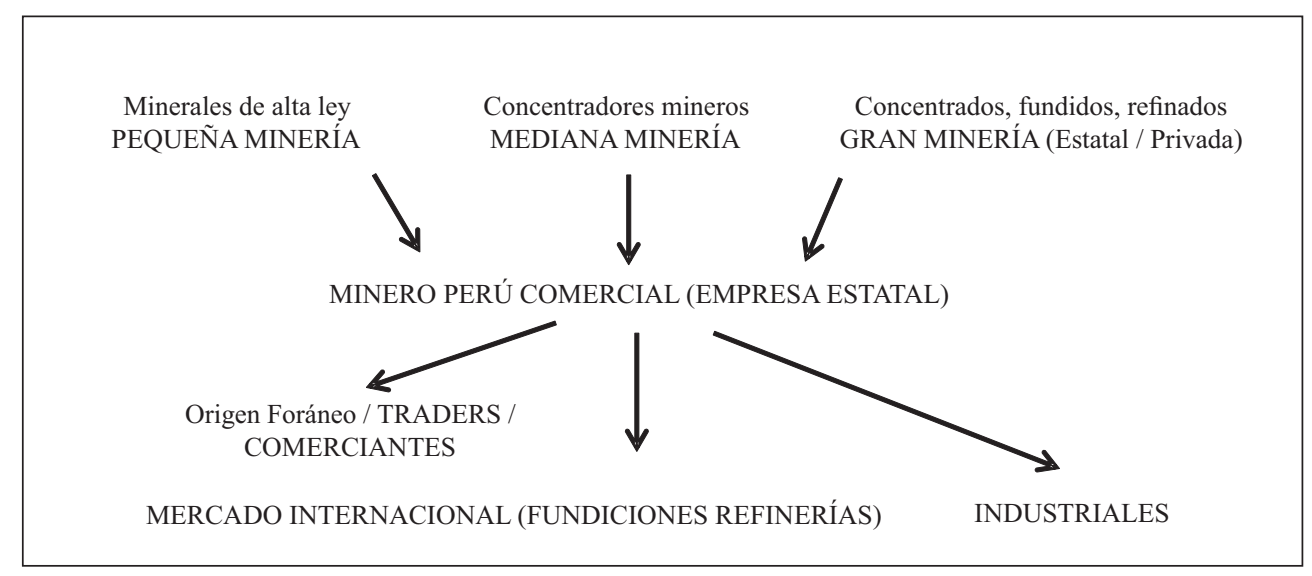




\section{Pensamiento Crítico Vol.I7. No I}

Durante este periodo la actividad minera se reglamentó a través de la Ley General de Minería 18880 (1970). La que posteriormente se vió modificada con el Decreto Supremo N. ${ }^{\circ}$ 020- MEM (1971), por el cual se aprueba el reglamento referente a la comercialización de los Productos minero metalúrgicos.

El espíritu de estas leyes interpretaba tanto el ideario como papel del gobierno de la Junta Militar, que con carácter nacionalista, estatista, así como de propulsor y regulador, modifica de manera sustantiva la legislación que hasta la fecha venía normando la actividad minera y su comercialización.

Se aprecia en el flujograma anterior que la participación del estado se hace manifiesta al interpretar por intermedio de la Empresa Pública Minero Perú Comercial (MINPECO), el rol de monopolio de la actividad comercializadora de la producción minera y metalúrgica nacional tanto de las empresas privadas, las nacionalizadas y las de reciente creación de carácter estatal ${ }^{2}$.

Dado el carácter monopólico de la empresa estatal "Minpeco", se logró controlar la subvaluación y sobrevaloración en la comercialización de la producción minera, situación que contribuyó de manera eficaz a la generación de una tributación consistente y coherente con el real valor de la producción minera exportable.

Así mismo, se logró transferir a los productores mineros principalmente de la Mediana y Pequeña minería, las condiciones más favorables que ofrecía el mercado en esos momentos, con lo cual se permitió el crecimiento de este sub-sector productivo.

Se posibilitó, el crecimiento de la Pequeña minería que producía productos mineros con márgenes de contenidos contaminantes, mediante la implementación de mezclas con valor comercial.

Se generó lo que podemos llamar, "El poder de negociación del Estado", en actividades como en la negociación de los fletes marítimos, seguros, formación de marca y precios nacionales con carácter y vigencia internacional.

2 El Gobierno de la Junta Militar, nacionaliza la Cerro de Pasco Corporation (1974) y la Marcona Mining Company (1975) y las convierte en las Peruanas Centromin Perú y Hierro Perú respectivamente. 


\section{Juan José Ospino Edery}

Minero Perú Producción contribuyó al financiamiento y construcción de las refinerías de Ilo para el cobre blíster producido por la fundición en la Southern en la misma localidad y la refinería de zinc en Cajamarquilla, cuyo pago se garantizaba con las ganancias obtenidas por la comercialización por parte de Minpeco. Aquí cabe añadir que las dos refinerías mencionadas son las únicas de envergadura construidas en los últimos 35 años.

\section{Estructura de la Comercialización de la Producción Minero Metalúrgica, Periodo 1980-1990.}

Flujograma de la Comercialización de la Producción Minero Metalúrgica

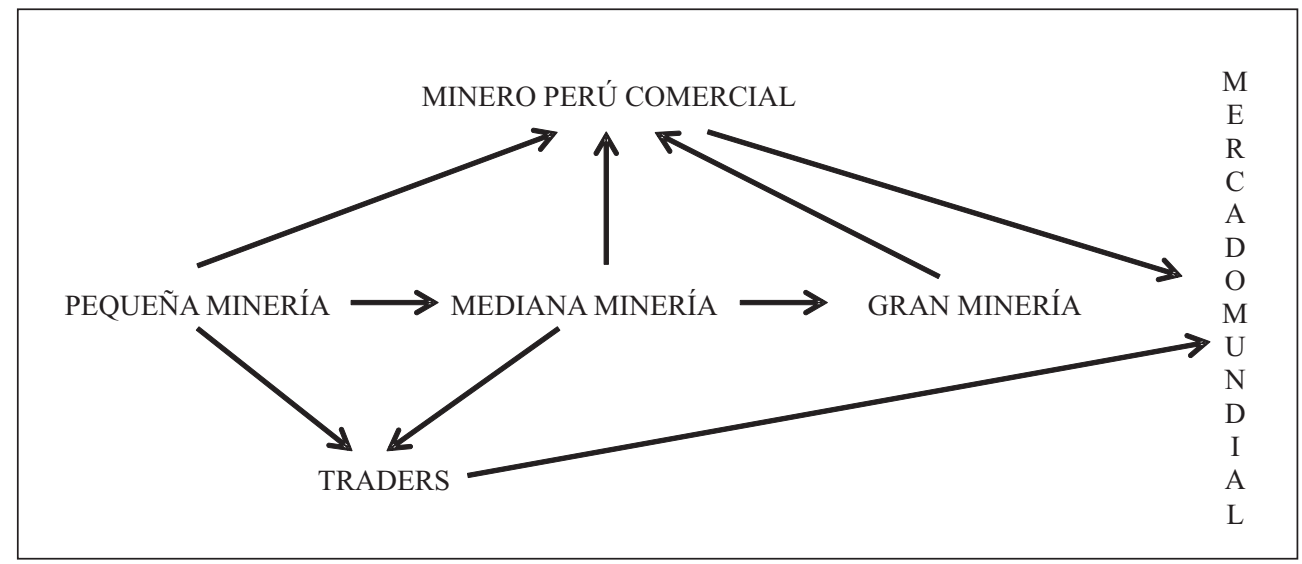

Este periodo abarca tanto, el segundo gobierno del Presidente Fernando Belaunde como el primer gobierno del Presidente Alan García.

Sea aprecia en el flujograma anterior lo siguiente:

Un cambio en el mismo como resultado de la implementación del DL 109 dado durante el gobierno del Presidente Belaunde, el cual indicaba que la actividad comercial de la producción minera podía ser realizada por agentes privados (traders/comerciantes), solo de las empresas de la mediana y pequeña minería en competencia con la empresa estatal "Minpeco", reservándose para el estado la comercialización de las empresas de Gran Minería estatal y privada. 
El esquema planteado resultó muy favorable para el productor minero, dado que la situación trazada, permitía la competencia por mejores condiciones transferidas a los actores de la producción, así como de instrumento de control y regulador para una real valorización de la producción minera y metalúrgica.

\section{Estructura de la Comercialización de la Producción Minero Metalúrgica, Periodo 1990 a la fecha}

Flujograma de la Comercialización de la Producción Minero Metalúrgica

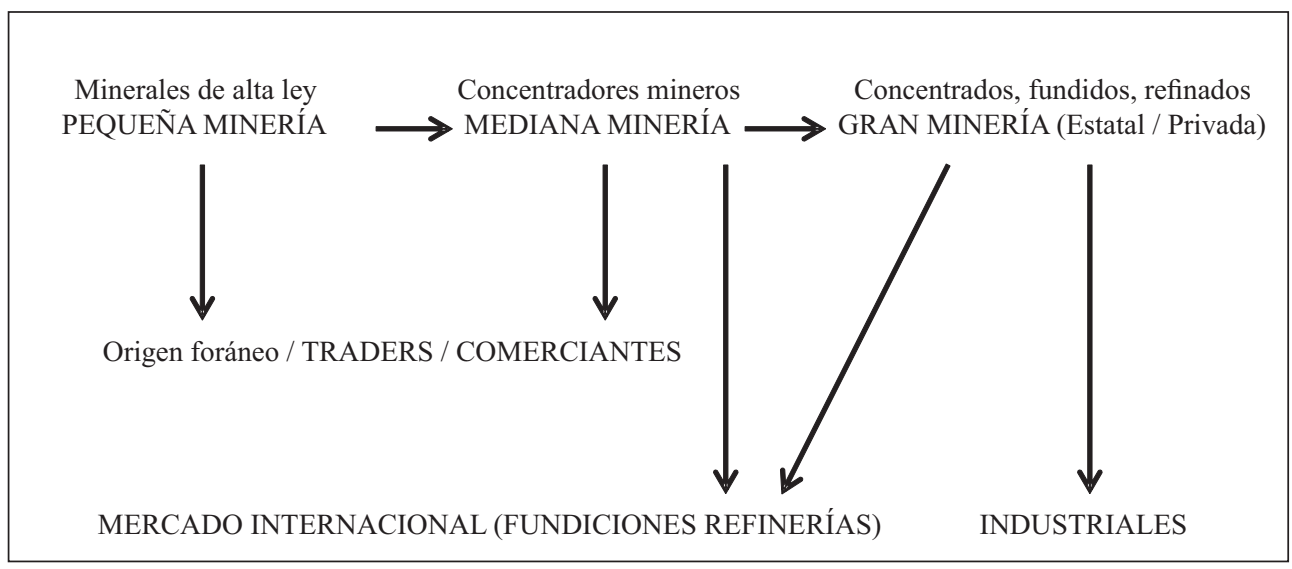

Este periodo se caracteriza por la privatización de la comercialización y desaparición de la empresa pública "Minpeco", con lo cual se acaba con el rol de regulador y fiscalizador que venía llevando a cabo esta organización.

Se regresa a los esquemas imperantes en la década de los años cincuenta, donde el estado es solo un espectador de tan importante actividad como es la comercialización de la producción minera que es el fin de toda inversión en este sector.

Cabe resaltar que hoy no existe por parte del estado ninguna organización que fiscalice esta actividad la cual es la más trascendental de la actividad minero metalúrgica. 


\section{Juan José Ospino Edery}

\section{Incidencia de la actividad minero metalúrgica y su comercialización en la economía del Perú.}

Es conocido el aporte de la actividad minera a la economía de nuestro país, el cual contribuye con alrededor del $60 \%$ del valor total exportado Cuadro N. ${ }^{\circ} 1$, lo que también nos señala cuan dependiente y vulnerable es la misma del mercado internacional, aspecto que debe ser de preocupación constante afín de potenciar otros sectores económicos con la finalidad de restarle preponderancia a esa dependencia.

\section{Cuadro N. $^{\circ} 1$}

Total de Exportaciones (porcentajes)

\begin{tabular}{|l|c|c|c|c|c|c|c|c|c|c|c|}
\hline Sectores & $\mathbf{1 9 9 6}$ & $\mathbf{1 9 9 7}$ & $\mathbf{1 9 9 8}$ & $\mathbf{2 0 0 0}$ & $\mathbf{2 0 0 2}$ & $\mathbf{2 0 0 3}$ & $\mathbf{2 0 0 4}$ & $\mathbf{2 0 0 5}$ & $\mathbf{2 0 0 6}$ & $\mathbf{2 0 0 8}$ & $\mathbf{2 0 1 0}$ \\
\hline Pesqueros & 15.5 & 16.5 & 7.1 & 13.7 & 11.6 & 9.0 & 8.6 & 7.5 & 5.6 & 5.8 & 5.3 \\
\hline Agrícolas & 5.1 & 6.9 & 5.6 & 3.6 & 2.8 & 2.5 & 2.5 & 1.9 & 2.4 & 2.2 & 2.7 \\
\hline Mineros & $\mathbf{4 5 . 2}$ & $\mathbf{4 0 . 0}$ & $\mathbf{4 7 . 7}$ & $\mathbf{4 6 . 3}$ & $\mathbf{4 9 . 4}$ & $\mathbf{5 1 . 6}$ & $\mathbf{5 5 . 6}$ & $\mathbf{5 6 . 3}$ & $\mathbf{6 1 . 8}$ & $\mathbf{5 8 . 4}$ & $\mathbf{6 1 . 1}$ \\
\hline Petr y Deri & 6.0 & 5.5 & 4.0 & 5.5 & 6.8 & 6.8 & 5.0 & 8.8 & 7.4 & 8.6 & 8.7 \\
\hline No Tradici & 27.0 & 30 & 34.2 & 29.4 & 29.2 & 28.8 & 27.2 & 24.7 & 22.1 & 24.4 & 21.5 \\
\hline Otros & 1.2 & 1.1 & 1.4 & 1.5 & 1.2 & 1.3 & 1.1 & 0.8 & 0.7 & 0.6 & 0.7 \\
\hline Total & $\mathbf{1 0 0 . 0}$ & $\mathbf{1 0 0 . 0}$ & $\mathbf{1 0 0 . 0}$ & $\mathbf{1 0 0 . 0}$ & $\mathbf{1 0 0 . 0}$ & $\mathbf{1 0 0 . 0}$ & $\mathbf{1 0 0 . 0}$ & $\mathbf{1 0 0 . 0}$ & $\mathbf{1 0 0 . 0}$ & $\mathbf{1 0 0 . 0}$ & $\mathbf{1 0 0 . 0}$ \\
\hline
\end{tabular}

Fuente: BCRP. SUNAT. INEI. Compendio estadístico 2011.

En el último lustro se ha registrado un aumento excepcional por ingreso de exportaciones de la producción minero metalúrgica, lo cual se atribuye a la mejora sustantiva de los precios de los metales a escala internacional lo cual se aprecia en el Cuadro $\mathrm{N} .^{\circ} 2$, lo que ha inducido probablemente a la realización de producciones marginales, lográndose un incremento de los volúmenes comercializados. 


\section{Cuadro Nro.2}

Cotización Internacional de Productos Mineros

\begin{tabular}{|l|c|c|c|c|c|c|c|}
\hline Productos & $\mathbf{2 0 0 4}$ & $\mathbf{2 0 0 5}$ & $\mathbf{2 0 0 6}$ & $\mathbf{2 0 0 7}$ & $\mathbf{2 0 0 8}$ & $\mathbf{2 0 0 9}$ & $\mathbf{2 0 1 0}$ \\
\hline Cobre (Londres) c/US\$/lb & 129.99 & 166.87 & 304.91 & 322.93 & 315.51 & 233.52 & 342.28 \\
\hline $\begin{array}{l}\text { Plata (Harman) US\$/ } \\
\text { OnzTroy }\end{array}$ & 6.69 & 7.34 & 11.57 & 13.42 & 15.01 & 14.68 & 20.19 \\
\hline Plomo (Londres)c/US $\$ / \mathrm{Lb}$ & 40.21 & 44.29 & 58.50 & 117.03 & 94.83 & 77.91 & 97.61 \\
\hline Zinc (Londres) c/US/lb & 47.53 & 62.68 & 148.56 & 147.07 & 85.04 & 75.05 & 96.18 \\
\hline Oro (Londres) US\$/ OnzTroy & 409.85 & 445.47 & 604.58 & 697.41 & 872.72 & 973.62 & 1225.29 \\
\hline
\end{tabular}

Nota: La cotización de productos mineros es sobre el valor CIF. Promedio del Periodo. Fuente INEI 2011.

La contribución del sector minero a las exportaciones ocurre, principalmente por la venta de la producción en forma de concentrados tal como se puede apreciar en el Cuadro $\mathrm{N}^{\circ}$ 3, donde los más representativos son los concentrados de Zinc y concentrados de Cobre ${ }^{3}$.

Como es de conocimiento, el concentrado minero, es producto de una etapa intermedia de valor agregado, completándose esta con la refinación, como etapa final previa al inicio de los procesos de industrialización del metal.

3 Los mayores volúmenes de exportación minera metalúrgica en el Perú se dan bajo la forma de concentrados dado que la capacidad de fundición y refinación se ve limitada a que la refinería de Cajamarquilla para el caso del zinc que trata una parte de la producción de concentrados de este metal, quedando volúmenes importantes como excedentes para el mercado externo, similar situación se presenta para los concentrados de cobre, en este caso la fundición de Ilo solo refina el cobre blíster de los concentrados que produce la Southern de sus minas Toquepala y Cuajone. Cabe resaltar que la paralización de la Fundición de la Oroya (Administrada por la empresa Doe Run) no ha permitido una mayor demanda interna de concentrados de zinc y cobre al estar paralizada la fundición y refinería. 
Juan José Ospino Edery

Cuadro Nro. 3

Perú: producción de concentrados

\begin{tabular}{|l|c|c|}
\hline Años & Miles TMF Zn & Miles TMF Cu \\
\hline $\mathbf{2 0 0 0}$ & 910.0 & 554.0 \\
\hline $\mathbf{2 0 0 5}$ & 1201.7 & 1009.8 \\
\hline $\mathbf{2 0 0 6}$ & 1203.4 & 1048.5 \\
\hline $\mathbf{2 0 0 7}$ & 1444.4 & 1190.3 \\
\hline $\mathbf{2 0 0 8}$ & 1602.6 & 1267.9 \\
\hline $\mathbf{2 0 0 9}$ & 1512.9 & 1276.3 \\
\hline $\mathbf{2 0 1 0}$ & 1470.5 & 1247.2 \\
\hline $\mathbf{2 0 1 1 e}$ & 1255.9 & 1235.2 \\
\hline
\end{tabular}

Fuente: SUNAD/SUNAT/ Ministerio de Energía y Minas. (e).

El cuadro siguiente $\mathrm{N} .{ }^{\circ} 4$, nos presenta un balance de minas y refinerías de concentrados por regiones geográficas. El Perú, para el caso de las exportaciones de concentrados de zinc, ocupa entre un tercer o cuarto puesto a escala mundial y dado que no cuenta con las refinerías suficientes para procesar (refinar), el volumen de concentrados que produce, nos coloca en una posición de dependencia frente a las refinerías foráneas.

Son las fundiciones y refinerías las que logran con su posición en el mercado definir las condiciones de refinación afectando la valorización de los concentrados que adquieren, las mismas que son susceptibles de ser manipuladas.

Cuadro N. ${ }^{\circ} 4$

Minas y refinerías de Zinc

\begin{tabular}{|l|c|c|}
\hline \multicolumn{1}{|c|}{ Ubicación } & Minas & Refinerías \\
\hline África & 6 & 3 \\
\hline Asia & 19 & 46 \\
\hline Australia & 9 & 3 \\
\hline Latinoamericana & 64 & 7 \\
\hline
\end{tabular}




\section{Pensamiento Crítico Vol.I7. No I}

\begin{tabular}{|c|c|c|}
\hline Norteamérica & 15 & 9 \\
\hline Europa & 12 & 28 \\
\hline TOTAL & 125 & 96 \\
\hline
\end{tabular}

Las más representativas. Fuente: ILZSG

\section{Conclusiones}

El estado peruano a la fecha no tiene mayor control sobre el real valor de las exportaciones de concentrados, con lo cual posibilita la práctica de la sobrevalorización y subvaluación (transferencia de precios) por lo cual urge la conformación de alguna forma de organización estatal que perfeccione dicho control.

El mercado de concentrados para nuestro país resulta el de mayor envergadura dado que bajo esta calidad son los mayores volúmenes que exportamos a fundiciones y refinerías que son en número mucho menor que los compradores de refinados (industriales).

Comercialización de la producción minero metalúrgica se constituye en la razón del negocio de la inversión minera.

El LME y el COMEX, así como las publicaciones especializadas Metals Week y Metal Bulletin, se constituyen en organismos donde la formulación del precio de los metales se da por acción de operaciones de compra-venta por un volumen anual pequeño comparado con el metal comercializado en el resto del mundo, no obstante sus cotizaciones son utilizadas por la gran mayoría de países, sin que estos realicen directamente transacciones en las Bolsas de Metales.

\section{Bibliografía}

Alfredo Dammert Lira: "Economía Minera" Minero Perú Comercial, 1990.

Juan José Ospino Edery. Pensamiento Crítico N. ${ }^{\circ}$ 8/ Enero del 2008, Revista del Instituto de Investigaciones Económicas de la Universidad Nacional Mayor de San Marcos. 


\section{Juan José Ospino Edery}

Latiza: “Asociación Latinoamericana de Zinc”. Estadísticas 1990-2000.

Memoria Anual del BCR: "Banco Central de Reserva del Perú", 2000- 2010.

Ministerio de Energía y Minas: Anuarios Mineros 1985-2010.

Miguel E Santillana Santos. La importancia de la actividad Minera en la Economía y la Sociedad. CEPAL 2006. 